\title{
Pulmonary artery catheter induced pulmonary artery rupture in patients undergoing cardiac surgery
}

Pulmonary artery rupture is a rare but rapidly fatal complication of pulmonary artery catheter insertion and use. Rapid diagnosis and appropriate treatment are essential to avoid mortality. Two cardiac surgical patients who suffered pulmonary artery rupture as a result of pulmonary artery catheter insertion and use are presented. These patients demonstrate two different presentations and the treatment of pulmonary artery rupture. A discussion of diagnosis, treatment, pathogenesis and prevention are presented.

\section{Key words}

COMPLICATIONS: pulmonary artery rupture; PULMONARY ARTERY CATHETER: pulmonary artery rupture; SURGERY: cardiac.

Common complications related to the insertion and use of the pulmonary artery catheter such as atrial premature beats, ventricular premature beats, transient right bundle branch block, and arterial puncture are minor and easily treatable. ${ }^{1,2}$ Pulmonary artery rupture is a rare but rapidly fatal complication of pulmonary artery catheter insertion and use, A 45 per cent fatality rate has been quoted with the time until death ranging from a few minutes

From the University of Michigan Medical Center, Department of Anesthesiology, Ann Arbor, Michigan.

Address correspondence to: Dr. Betty Jean Muller, Department of Anesthesiology, Room A6061, Box 043, University of Michigan Medical Center, 1405 E. Ann Street, Ann Arbor, Michigan 48109. to 24 hours. ${ }^{3,4}$ Most of these patients die of rapid, massive exsanguination.

Even with its rare occurrence most of the reported cases of pulmonary artery rupture caused by pulmonary artery catheters have occurred in the perioperative period, and the majority of these occurred in cardiac surgical patients. ${ }^{4-6}$ Despite this there have been few case reports in the anaesthesia literature and only two occurred in the perioperative period of noncardiac surgical patients. ${ }^{-10} \mathrm{We}$, therefore, present two cases of pulmonary artery rupture occurring in cardiac surgery patients which have required management by anaesthetists. These two cases demonstrate the difference that rapid diagnosis and appropriate treatment can have on patient outcome and duration of hospitalization. A discussion of diagnosis, treatment, pathogenesis and prevention follows.

\section{Case 1}

A 71-year-old female was admitted for a mitral valvulotomy and double coronary artery bypass grafting. Cardiac catheterization revealed significant mitral stenosis and occlusion of the obtuse marginal and right coronary arteries. Past medical history was significant for a 40 pack year smoking history and a stroke two years prior to admission without residual neurologic deficits.

The patient was brought to the operating room and two peripheral intravenous cannulae, a radial artery catheter, and a pulmonary artery catheter via the right internal jugular vein were inserted with ease. The pulmonary artery pressure was $37 / 19 \mathrm{mmHg}$. The pulmonary artery catheter provided a consistently clear pulmonary artery waveform prior to 
cardiopulmonary bypass. As soon as pulmonary blood flow was reestablished prior to the termination of cardiopulmonary bypass, a damped pulmonary artery tracing was noted. The catheter was flushed twice without restoration of a clear waveform and withdrawal of the catheter was attempted. Considerable force was required to withdraw the catheter the $2 \mathrm{~cm}$ needed to restore a pulmonary artery waveform. The patient required a dopamine infusion at the termination of cardiopulmonary bypass. Approximately one hour after repositioning the catheter, blood was seen in the endotracheal tube. It was suctioned and $20 \mathrm{~mL}$ of blood and clot was removed. This was attributed to local trauma by the endotracheal tube. The patient was being ventilated with 100 per cent oxygen; however, arterial blood gases at this time revealed a $\mathrm{PaO}_{2}$ of $7.87 \mathrm{kPa}$ (59 torr). Prior to bypass the $\mathrm{PaO}_{2}$ on 100 per cent oxygen ranged from 78.4 to $59.7 \mathrm{kPa}$ ( 588 to 488 torr). Ten centimeters of positive end-expiratory pressure (PEEP) were added with improvement of oxygenation to $16.4 \mathrm{kPa}$ (123 torr). Subsequent suctioning of the endotracheal tube returned no more blood. A chest $x$-ray obtained immediately after transfer to the intensive care unit was read as bilateral pulmonary infiltrates, greater on the right than on the left. The patient was treated with diuretics and a repeat chest $x$-ray three hours later showed residual right pulmonary infiltrates (Figure 1). As the patient's $\mathrm{PaO}_{2}$ began to improve the PEEP was decreased from 10 to $3 \mathrm{~cm}$ over eight hours.

The following evening (33 hours post-op) the endotracheal tube was suctioned and retumed a moderate amount of bright red blood. Massive haemorrhage into the right chest tube followed. One thousand $\mathrm{mL}$ of blood drained from the right chest tube in two to three minutes. The systolic blood pressure fell rapidly from 120 to $50 \mathrm{mmHg}$. The median sternotomy incision was opened and no mediastinal or cardiac bleeding was found. The right pleural cavity was explored and the right lower lobe pulmonary artery was found to be ruptured just as it entered the lung parenchyma. Several sutures were placed to control the bleeding. Over the two hours it took to stabilize the patient she bled approximately $6,700 \mathrm{~mL}$ and was replaced with 15 units of blood. The patient was then returned to the operating room for further exploration and repair of the pulmonary artery laceration. Postoperatively,

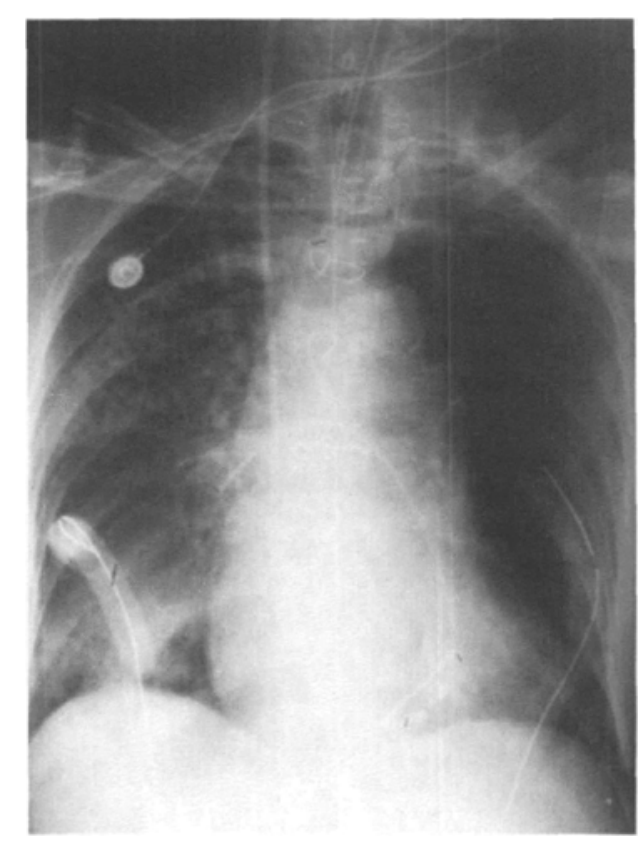

FIGURE 1 Patient 1 . Chest $x$-ray taken three hours after entry into the ICU. Pulmonary infiltrates can be seen on the right.

haemoptysis continued for one week. A right lower lobe infiltrate was observed on chest $x$-ray for three weeks. The patient's postoperative course was complicated by sepsis, acute renal failure, and respiratory failure requiring prolonged ventilation. These problems gradually resolved but the patient was left with a severe hearing loss felt to be caused by aminoglycoside antibiotics and furosemide. The patient was discharged from the hospital in good condition, with the exception of her hearing loss, 45 days after the initial operation.

\section{Case 2}

An 87-year-old female was admitted for aortic valve replacement. Cardiac catheterization revealed moderate aortic stenosis, a pulmonary artery pressure of $45 / 17 \mathrm{mmHg}$, and a cardiac output of $2.631 \cdot \mathrm{min}^{-1}$. Other medical problems included hypertension and a stroke eight years prior to admission with residual left hand paresis.

Prior to operation a pulmonary artery catheter was inserted through the right internal jugular vein. 


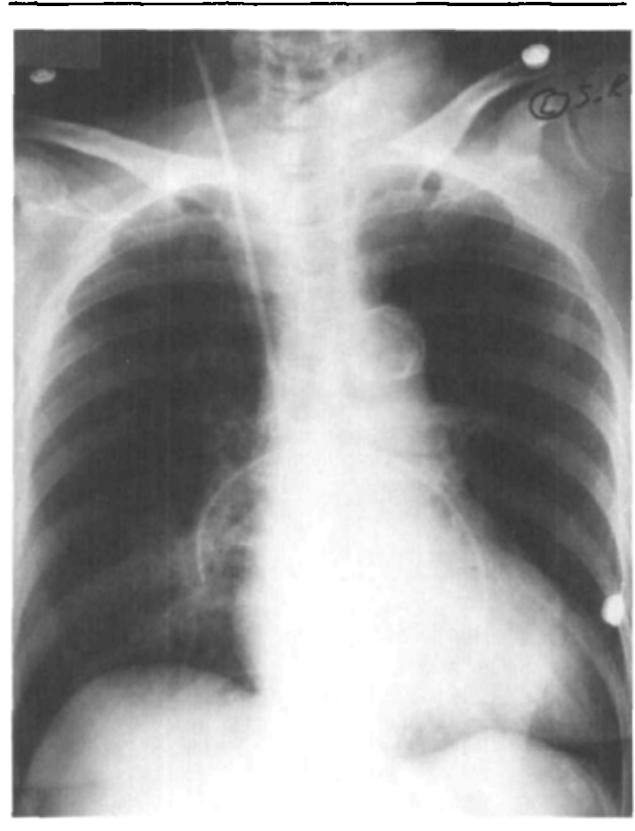

FIGURE 2 Patient 2. Chest $x$-ray taken immediately after haemoptysis. An infiltrate can be seen surrounding the tip of the peripherally located pulmonary anery catheter.

The pulmonary artery was entered at $45 \mathrm{~cm}$ of catheter length but difficulty was experienced in obtaining a wedge tracing. The catheter was withdrawn and advanced several times. At one point the catheter was advanced to $65 \mathrm{~cm}$ of its length. After successfully obtaining a wedge tracing, the patient began coughing incessantly. Approximately $30 \mathrm{~mL}$ of bright red blood was suctioned from her mouth. A chest $x$-ray showed an infiltrate surrounding the catheter tip in the right lower lobe of the lung (Figure 2). The patient exhibited a decrease in blood oxygenation at this time. Before this event while receiving two liters of oxygen by nasal cannulae the $\mathrm{PaO}_{2}$ was $12.9 \mathrm{kPa}$ (97 torr). After haemoptysis, while breathing 100 per cent oxygen by mask, the $\mathrm{PaO}_{2}$ was $8.5 \mathrm{kPa}$ (64 torr). The operation was cancelled, the catheter removed, and the patient was transferred to the intensive care unit, in the right lateral decubitus position. Five hours afterward, the patient's $\mathrm{PaO}_{2}$ began to improve. It was $14.67 \mathrm{kPa}$ (110 torr) on two liters of oxygen by nasal cannulae and remained adequate thereafter. Haemoptysis continued for four days and there was resolution of the right lung infiltrate on chest $x$-ray during that same interval. Seven days after the incident the patient returned to the operating room for aortic valve replacement. Pulmonary artery catheter insertion was easily accomplished. An uneventful surgical procedure ensued, and the patient was discharged from the hospital 17 days after admission.

\section{Discussion}

\section{Diagnosis}

Haemoptysis is the hallmark of catheter induced pulmonary artery rupture..$^{4.11-13}$ It may present as small amounts of blood or as massive exsanguination leading to rapid death. The blood is usually bright red but both poorly and well oxygenated blood have been observed in well documented cases. Other signs of pulmonary artery rupture include: hypotension, a falling haematocrit, dccreased $\mathrm{PaO}_{2}$, dyspnoea, bronchospasm, pleural effusion, and pneumothorax. ${ }^{4,13-15}$ Perforation of the pulmonary artery catheter tip into a bronchus may result in the aspiration of air through the distal lumen. ${ }^{16}$

Despite the often catastrophic presentation of pulmonary artery perforation, some less severe cases may not initially be correctly diagnosed. The differential diagnosis includes pulmonary oedema, pulmonary infarction, and trauma of the tracheobronchial tree. Differentiation of pulmonary infarction from pulmonary artery rupture can be difficult but carries great therapeutic and prognostic importance.

If time allows, there are several procedures useful in diagnosing pulmonary artery rupture. A chest $x$-ray will show a new parencnymal infiltrate around the catheter tip. This will demonstrate the extent and area of involvement, usually in the right middle or lower lobes. ${ }^{17} \mathrm{~A}$ pleural effusion or pneumothorax may also be seen or may mask the presence of an infiltrate. Injection of radiographic contrast material through the distal lumen of the catheter followed immediately by a chest $x$-ray may show contrast material in the tracheobronchial tree. ${ }^{12} \mathrm{~A}$ radiograph taken two minutes after dye injection may show extravasation of contrast material into the pulmonary interstitium. ${ }^{5}$ Bronchoscopy may be useful to locate the involved area and observe the degree of haemorrhage. It must be 
stressed that diagnostic procedures should be selected with full regard to the urgency of definitive treatment.

\section{Treatment}

Treatment depends upon the degree of haemorrhage exhibited and the stability of the patient's clinical status. The pulmonary artery catheter should be withdrawn into a large proximal artery. ${ }^{6}$ In mild cases this may be sufficient to effect a cessation of bleeding. The affected side should be positioned down to prevent blood from entering the unaffected lung. ${ }^{9,13}$ If an operative procedure was planned, it should be cancelled and supportive therapy of the patient begun. This includes oxygen and keeping coughing, airway suctioning, and straining to a minimum. Patients with elevated pulmonary artery pressures may need drug therapy to lower the pressure and reduce the amount of pulmonary artery bleeding. ${ }^{6}$ Any degree of anticoagulation should be reversed. If the patient has an endotracheal tube in place one report suggests that PEEP may be useful in controlling pulmonary artery haemorrhage. ${ }^{8}$ Another author suggests that an endotracheal tube be replaced by a double lumen endobronchial tube to allow for both evacuation of blood and protection and ventilation of the uninvolved lung. ${ }^{9}$ If a double lumen tube is placed, the unaffected lung will be protected from the spillage of blood from the affected side. In this situation the affected side may be positioned uppermost to utilize the gravity induced decrement in pulmonary artery blood flow to decrease the bleeding. ${ }^{8}$

Any patient who has suffered pulmonary artery perforation should be observed in an intensive care unit for at least 24 hours to allow for rapid diagnosis and treatment in the event of rebleeding. ${ }^{6}$ Pulmonary artery pressure, arterial blood gases, and serial chest $x$-ray should be monitored. The appropriate time for rescheduling an elective operative procedure has not been established firmly, but as a minimum arterial blood gases should have returned to normal, haemoptysis should have ceased, and the infiltrate on chest $x$-ray should have cleared.

Severe pulmonary artery haemorrhage requires rapid surgical control of the bleeding. ${ }^{4,6}$ If pulmonary artery haemorrhage is observed at the end of cardiopulmonary bypass, resumption of bypass will allow for unhurried location and correction of the pulmonary artery laceration.
Depending on the site and extent of damage, this may require suturing of a pulmonary artery, resection of a lobe of the lung, or possibly even pneumonectomy. If pulmonary artery haemorrhage begins postoperatively, the severity of the bleeding, either as haemoptysis or from a chest tube, will dictate whether the patient returns to the operating room or whether the chest must be opened in the intensive care unit. The right pulmonary artery tree is more often the site of haemorrhage and should be explored first. ${ }^{13}$ Airway maintenance and effective ventilation in the face of severe haemoptysis may be a problem. Positive end-expiratory pressure may improve oxygenation and ventilation. ${ }^{8}$ If the clinical circumstances allow it, placement of a double lumen endobronchial tube will permit isolation and ventilation of the unaffected lung from the side involved. Technical difficulties imposed by rapid haemorrhage into the trachea and larynx may preclude double lumen endobronchial tube placement. In this situation immediate surgical control of the bleeding is the only solution.

\section{Pathogenesis}

A number of mechanisms, both with and without experimental substantiation, have been proposed to explain the occurrence of pulmonary artery rupture. Based on the clinical circumstances of early case reports, speculative mechanisms for catheterinduced pulmonary artery rupture were proposed. Clinical and laboratory studies in recent years have begun to clarify the situation.

Shin, studying 60 intensive care unit patients, injected radiographic contrast dye into the balloon port of a previously inserted pulmonary artery catheter. ${ }^{18}$ Fifteen catheters demonstrated eccentric inflation. This can leave the catheter tip exposed beyond the balloon and makes it possible for the tip to perforate through the vessel wall. These eccentrically inflated catheters were located far peripherally. One of these patients developed haemoptysis after repeated measurements. Barash performed a similar study in human cadaver lungs. ${ }^{5}$ He inflated the balloon with radiographic contrast and demonstrated the occurrence of eccentric inflation. Dissection of the lungs demonstrated three mechanisms for vascular perforation: (1) balloon disruption, (2) distorted balloon inflation causing the catheter tip to be propelled through the vessel wall, (3) catheter tip advancement far 
peripherally with subsequent perforation of the vessel.

McDonald investigated the pressure-volume relationship of the pulmonary artery catheter balloon and found that pressures greater than $500 \mathrm{mmHg}$ were generated in the balloon by inflation. ${ }^{19}$ After inflation the pressure within the balloon fell to $288 \pm 29.9 \mathrm{mmHg}$ and remained the same with progressive inflation of the balloon with up to $1.5 \mathrm{~mL}$ of air.

Hardy studied the pressure required to rupture pulmonary arteries. ${ }^{3}$ In human cadaver lungs 2989 $\pm 1109 \mathrm{mmHg}$ was required to rupture distal pulmonary arteries while $2040 \pm 560 \mathrm{mmHg}$ pressure was required to rupture mid-pulmonary arteries. These pressures were obtained with fluid filling of the balloon. When catheter balloons were inflated with $1.5 \mathrm{~mL}$ of air the maximal pressure exerted on a distal pulmonary artery was $1686 \pm$ $151 \mathrm{mmHg}$ and two pulmonary artery ruptures occurred ( $n=14$ ). In mid-pulmonary arteries $1.5 \mathrm{~mL}$ of air generated a maximal pressure of 1091 $\pm 232 \mathrm{mmHg}$ and three pulmonary artery ruptures $(n=20)$. Direct observation of the balloon in a distal pulmonary artery showed glove-like filling of pulmonary artery branches with rupture occurring with lateral bulging of the balloon. Histologic study of pulmonary arteries that had been stressed by $1400 \mathrm{mmHg}$ intraluminal pressure without rupturing showed no structural changes in the arterial wall.

The data relating to the pressure required to rupture 14 distal pulmonary arteries and 20 mid-pulmonary arteries were statistically analyzed with reference to (1) age and (2) evidence of pulmonary artery hypertension. In these cadaver lungs the description of pulmonary arterial atheroma on autopsy report was taken as evidence of pulmonary hypertension. The statistical analysis showed that age greater than 60 years increased the risk of pulmonary artery rupture while the existence of pulmonary hypertension did not.

Many clinical case reports state that the existence of pulmonary artery hypertension places the patient at higher risk for pulmonary artery rupture. ${ }^{4-6,10,13.14}$ This is based on the observation that many patients in whom pulmonary rupture occurred also had pulmonary artery hypertension. The apparent discrepancy between this experimental evidence and the clinical observations may be related to several facts. The histological changes of pulmonary hypertension are minimal in the distal or midportions of pulmonary arteries which were the areas tested in the study. ${ }^{3}$ The most prominent changes are found in larger pulmonary arteries. Pulmonary hypertension may be associated with a more distal position of the catheter and lead to vessel rupture in too peripheral a location. It has been pointed out that clinically it is impossible to distinguish whether pulmonary hypertension predisposes to pulmonary artery rupture or whether it is one of several characteristics of the population in which this procedure is performed. ${ }^{13}$ Thus the relationship of pulmonary hypertension to pulmonary artery rupture must be questioned.

\section{Prevention}

The above studies delineate advanced age (greater than 60 years) and peripheral location of the pulmonary artery catheter as two factors associated with an increased likelihood of pulmonary artery rupture. To avoid peripheral location of the catheter it has been suggested that the tip of the pulmonary artery catheter should not be advanced more than $4-5 \mathrm{~cm}$ beyond the pulmonary valve or more than $5 \mathrm{~cm}$ from the mediastinum on chest $x$-ray., ${ }^{4,5}$ The first author recommends using a protective sheath around the catheter so that it can be further advanced if wedge pressures are needed and emphasizes the importance of obtaining wedge pressures only when needed. It is also recommended that $1.5 \mathrm{~mL}$ of air be placed in the balloon during insertion to prevent it from locating peripherally. Careful observation of the pressure tracing during insertion is necessary as unrecognized $V$ waves in the wedge tracing may lead to further advancement of the catheter into a far peripheral location.

The balloon pressure studies emphasize that the balloon should not be inflated against undue resistance. McDonald further suggests locating the catheter in a yessel where wedge pressure tracings can be obtained only by inflating the balloon beyond the point of instantaneous loss of resistance, the time of greatest intraballoon pressure. ${ }^{19}$ This will prevent the high pressure impact of balloon inflation being transmitted to the vessel wall. At the same time, excessive balloon inflation should be avoided. Gradual inflation of the balloon until a wedge pattern is observed is preferable to injection of a 
standard volume of air. If an "overwedge" pattern is obtained with balloon inflation, the catheter should be withdrawn since this results from impingement of the catheter against the vessel wall or herniation of the balloon over the catheter tip. ${ }^{5}$

Even when pulmonary artery catheters have been positioned properly, they may migrate out further in the pulmonary vasculature. Several mechanisms have been proposed: the normal pulsation of blood through the heart and vasculature, lung volume changes, postural changes of the patient, peripheral migration of a catheter loop in the right ventricle, and forced fluid injection for cardiac output determination. This potential for peripheral migration of the catheter requires constant vigilance by the anaesthetist. When a smaller volume of air in the balloon produces a wedge tracing, the catheter should be withdrawn. Any evidence of a redundant loop of catheter in the right ventricle should lead to repositioning of the catheter. Cardiac outputs should not be performed when it is evident that the catheter has migrated peripherally or when an "overwedge" tracing has been obtained as the force fluid injection may drive the catheter tip even farther out into the periphery or against the vessel wall.

Events occurring during cardiac surgery have been suggested as contributing to pulmonary artery perforation and bleeding. They are intraoperative manipulation of the heart, hypothermia causing catheter stiffening, entrapment of the catheter by a superior vena caval snare with subsequent manipulation of the empty heart, and systemic anticoagulation which will promote bleeding. Indeed the majority of case reports of pulmonary artery rupture have been in cardiac surgical patients. It has been recommended that pulmonary artery catheters be pulled back up to $5 \mathrm{~cm}$ prior to the establishment of cardiopulmonary bypass.

\section{Conclusions}

The first patient presented here demonstrated several signs of pulmonary artery rupture, endobronchial haemorrhage, hypotension, and a decrease in $\mathrm{PaO}_{2}$. These in combination with the force required to withdraw the peripherally placed pulmonary artery catheter should have raised the question of pulmonary artery rupture. All this occurred just after bypass when many of the factors considered to promote pulmonary artery rupture during cardiac surgery were present. These are hypothermia, manipulation of the heart, and anticoagulation. The force required to withdraw the pulmonary artery catheter may have been related to entrapment of the catheter by the superior vena caval snare. Perhaps the application of $10 \mathrm{~cm}$ of PEEP slowed or stopped further bleeding allowing the patient's condition to stabilize. The correct diagnosis was not made even after the chest $x$-ray obtained in the ICU showed right sided pulmonary infiltrates. PEEP was rapidly lowered and airway suctioning 33 hours later precipitated massive bleeding. Fortunately the diagnosis was made at this time and the patient was treated appropriately. Her near death postoperatively may have been avoided if the correct diagnosis had been considered and her chest had been explored intraoperatively during the first episode.

The second patient's age of 86 placed her at increased risk for pulmonary artery perforation. ${ }^{3}$ She probably suffered pulmonary artery rupture on the basis of the peripheral placement of the pulmonary artery catheter. In this case the diagnosis was made promptly and the patient was treated appropriately. The haemoptysis, decreased $\mathrm{PaO}_{2}$, and the infiltrate surrounding the catheter tip on the chest $x$-ray were all characteristic of pulmonary artery perforation. Cancellation of the operation removed added risks, especially anticoagulation which could have made the patient's condition much worse. She was appropriately observed and managed in an intensive care unit for 24 hours. The operation was rescheduled after the patient's symptoms, $\mathrm{PaO}_{2}$, and chest $x$-ray had returned to normal.

The incidence of pulmonary artery perforation by pulmonary artery catheters is low. One retrospective study of intraoperative pulmonary artery catheter use quoted an incidence of 0.2 per cent. ${ }^{6} \mathrm{~A}$ prospective intensive care unit study of 116 pulmonary artery catheter insertions reported none. ' Despite the low incidence the high potential for rapid mortality makes knowledge of the diagnosis and treatment essential. As demonstrated in these two cases rapid diagnosis and treatment can make a difference in patient outcome and the length of hospitalization. 


\section{References}

1 Elliot CG, Zimmerman GA, Clemmer TP. Complications of pulmonary artery catheterization in the care of critically ill patients. A prospective study. Chest 1979; 6: 647-52.

2 Katz JD, Cronau LH, Barash PG, Mandel SD. Pulmonary artery flow guided catheters in the perioperative period. JAMA 1977; 237: 2832-4.

3 Hardy $J-F$, Morissette $M$, Taillefer $J$, Vauclair $R$. Pathophysiology of rupture of the pulmonary artery by pulmonary artery balloon-tipped catheters. Anesth Analg 1983; 62: 925-30.

4 Kelly F, Jr., Morris GC, Crawford ES, Espada R, Howell JF. Perforation of the pulmonary artery with Swan-Ganz catheters. Diagnosis and surgical management. Ann Surg 1981; 293: 686-91.

5 Barash PG, Nardi D, Hammond G, et al. Catheterinduced pulmonary artery perforation. Mechanisms, management, and modifications. J Thorac Cardiovase Surg 1981; 82: 5-12.

6 McDaniel DD, Stone JF, Faltas $A N$, et al. Catheterinduced pulmonary artery hemornhage. Diagnosis and management in cardiac operations. J Thorac Cardiovasc Surg 1981; 82: 1-5.

7 Ohn KC, Cottrell JE, Turndorf H. Hemoptysis from a pulmonary-artery catheter. Anesthesiology 1979; 51: 485-6.

8 Scuderi PE, Prough DS, Price JD, Comer PB. Cessation of pulmonary artery catheter-induced endobronchial hemorrhage associated with the use of PEEP. Anesth Analg 1983; 62: 236-8.

9 Stein JM, Lisbon A. Pulmonary hemorrhage from pulmonary artery catheterization treated with endobronchial intubation. Anesthesiology 1981; 55: 698-9.

10 Krantz EM, Viljoen J. Haemoptysis following insertion of a Swan-Ganz catheter. Br J Anaesth 1979; 51: 457-9.

11 Golden MS, Pinder T, Jr., Anderson WT, Cheitlin $M D$. Fatal pulmonary hemorrhage complicating use of a flow-directed balloon-tipped catheter in a patient receiving anticoagulant therapy. Am J Cardiol 1981; 32: 865-86.

12 Rubin SA, Puckett RP. Pulmonary artery-bronchial fistula. A new complication of Swan-Ganz catheterization. Chest 1979; 75: 515-6.

13 Pautson DM. Scott STM, Sethi GK. Pulmonary hemorrhage associated with balloon flotation catheters. Report of a case and review of the literature. J. Thorac Cardiovsc Surg 1980; 80: 453-8.
14 Rosenbaum L, Rosenbaum SH, Askanazi J, Hyman $A I$. Small amounts of hemoptysis as an early warning sign of pulmonary artery rupture by a pulmonary artery catheter. Crit Care Med 1981; 9: 319-20.

15 Farber DL, Rose DM, Bassell GM, Eugene J. Hemoptysis and pneumothorax after removal of a persistently wedged pulmonary artery catheter. Crit Care Med 1981; 9: 494-5.

16 German JC, Allyn PA, Bartlett RH. Pulmonary artery pressure monitoring in acute burn management. Arch Surg 1973; 106: 788-91.

17 Benumof $J L$, Saidman $L$, Arkin DB, Diamant $M$. Where pulmonary artery catheters go: Intrathoracic distribution. Anesthesiology 1977; 46: 336-8.

18 Shin B, Ayella RJ, McAsian TC. Pitfalls of SwanGanz catheterization. Crit Care Med 1977; 5: 125-7.

$19 \mathrm{McD}$ onald $\mathrm{DH}$, Zaidan JR. Pressure-volume relationships of the pulmonary artery catheter balloon. Anesthesiology 1983; 59: 240-3.

\section{Résumé}

La rupture de l'artère pulmonaire est une complication rare mais rapidement fatale de l' insertion et l'utilisation d'un cathéter dans l'artère pulmonaire. Un diagnostic rapide et une thérapie appropriée sont essentiels afin d'éviter la mortalité. Deux patients se présentant pour chirurgie cardiaque et ayant souffert d'une rupture de l'artère pulmonaire comme résultat de l'insertion et de l'utilisation d' un cathéter dans l'artère pulmonaire sont discutés. Une discussion du diagnostic, du traitement, de la pathogenèse et de la prévention est présentée. 